\title{
Unrecognised, undertreated, pain in ICU: Causes, effects, and how to do better
}

\author{
S. M. Alderson, S. R. McKechnie
}

John Radcliffe Hospital, Oxford, UK

Email: s.m.alderson@gmail.com

Received 28 January 2013; revised 3 March 2013; accepted 12 March 2013

\begin{abstract}
Methods: a literature review from 1990 to August 2012. Introduction: pain and its recognition can be a particular problem for patients in intensive care units (ICUs). Studies have suggested that around $70 \%$ of ICU patients have unrecognised or undertreated pain. Pain has serious physical and psychological effects, and can impair patient recovery and discharge. Pain relief is also an ethical and professional responsibility of doctors and nurses-and we may be failing in this. Causes: pain may be due to medical and nursing procedures, and the ICU environment. Pain can be under-recognised because ICU patients are often impaired in their ability to communicate (e.g. secondary to confusion from acute illness, endotracheal intubation, or reduced conscious level from sedative agents). Tools for pain assessment: in patients able to communicate verbally, the Numerical Rating Scale (NRS) can be used to rate pain severity. In non-verbal, conscious, patients, the Visual Analogue Scale (VAS) can be used as a visual alternative. Both are well-established. For unconscious/sedated patients, the Behavioural Pain Scale (BPS) and Critical Care Pain Observation Tool (CPOT) have been developed and validated. Changes in practice: where possible, sedation practice can be changed to allow better recognition of pain. Constant deep sedation can be interrupted with daily "sedation holds" to allow pain assessment. "Analgo-sedation" may also be used, with drug regimes which prioritise analgesia over sedation. "No-sedation" approaches may also be considered, but further research is required.
\end{abstract}

Keywords: Pain; Pain Assessment; Pain Recognition; Pain Management; ICU; ITU; Intensive Care; Sedation; Analgo-Sedation

\section{INTRODUCTION}

Pain and its recognition can be a particular problem for patients in intensive care units (ICUs). Some studies have suggested that around $70 \%$ of ICU patients have unrecognised or undertreated pain [1]. Such pain is problematic because severe pain interferes with cardiovascular and respiratory physiology, and can therefore impair a patient's recovery and discharge [2]. Severe pain can also contribute to adverse psychological outcomes in ICU patients, including anxiety, depression, and post-traumatic stress disorder (PTSD) [3].

The relief of pain is a fundamental ethical and professsional responsibility for both doctors and nurses: our failure to recognise and treat pain may therefore be seen as an ethical and professional failing too $[4,5]$.

This article will review: 1) causes of pain on ICU and the issue of its under-recognition; 2) difficulties with pain assessment in ICU patients; 3) tools developed to improve pain assessment in ICU patients; 4) changes in ICU practice intended to improve pain assessment and management.

The medical management of pain falls outside of the scope of this review. A brief summary is presented in Table 1: the stepwise management of pain.

\section{CAUSES OF PAIN ON ICU AND ITS UNDER-RECOGNITION (TABLE 2)}

Pain is defined physiologically as an unpleasant sensory or emotional experience associated with actual or potential tissue damage [6]. Pain can also be defined as "whatever the experiencing person says it is, existing whenever he or she says that it does" [7].

ICU patients have many potential sources of pain. Clearly, acute pain may be caused by whatever injuries or illness required an ICU admission: for example, following trauma, surgery, or sepsis. However, pain may also be caused or worsened by routine medical procedures, nursing care, or by the ICU environment itself [2]. Many ICU patients also suffer from chronic pain prior to their ICU admission [8].

Medical procedures such as surgical incisions, drains, and the use of endotracheal tubes all have the potential to 
Table 1. The stepwise approach to pain management.

\begin{tabular}{|c|c|c|}
\hline & Description & Example \\
\hline 1 & Recognising pain & \\
\hline 2 & $\begin{array}{l}\text { Removing or modifying the } \\
\text { cause, if possible }\end{array}$ & Cooling a burn \\
\hline \multirow[t]{3}{*}{3} & Non-pharmacological therapies & $\begin{array}{l}\text { Reassurance, changing } \\
\text { patient position }\end{array}$ \\
\hline & Pharmacological therapies: & \\
\hline & Simple analgesia & Paracetamol, NSAIDs \\
\hline \multirow[t]{3}{*}{4} & Weak opiates & Tramadol \\
\hline & Strong opiates & Fentanyl, morphine \\
\hline & Adjuvant analgesics & Gabapetin \\
\hline 5 & Regional anaesthetic techniques & Epidurals \\
\hline 6 & Specialist review & Pain team \\
\hline
\end{tabular}

Table 2. Types and causes of pain in ICU.

\begin{tabular}{lll}
\hline Onset of pain & Type of pain & Examples of potential causes \\
\hline \multirow{2}{*}{ Acute } & $\begin{array}{l}\text { Bodily pain } \\
\text { (nociceptive/somatic) }\end{array}$ & $\begin{array}{l}\text { Fractured bone } \\
\text { Chest drain insertion }\end{array}$ \\
& $\begin{array}{l}\text { Nerve pain (neuropathic) } \\
\text { Bodily pain } \\
\text { (nociceptive/somatic) }\end{array}$ & Nerve compression \\
Chronic & Musculoskeletal back pain \\
& Nerve pain (neuropathic) & Sciatica \\
\hline
\end{tabular}

cause pain. Nursing procedures such as turning, tracheal suctioning, and dressing changes, may also cause pain. Acute confusional states or sleep deprivation associated with the ICU environment may also worsen a patient's experience of pain [2].

Although such potential sources of pain on ICU may appear obvious, studies have consistently demonstrated that we underestimate and undertreat pain in our patients. One study reported that $77 \%$ of patients recalled having experienced pain whilst on ICU; $32 \%$ of those reported their pain as severe and $60 \%$ reported their pain as moderate or severe [1]. This has lead one author to declare that we are simply not very good at assessing pain in ICU patients [9].

\section{PROBLEMS ASSESSING PAIN IN ICU PATIENTS}

Assessing pain in ICU patients can be more difficult than assessing pain in non-ICU patients [10]. For general hospital in-patients, the "gold-standard" of pain assessment is considered to be patient self-reporting: patients will usually tell us when they are in pain, and when they are not [11]. In ICU, however, a patient's ability to report their pain in this way is frequently impaired, either by their underlying illness or their treatment.

Patients in ICU may frequently be able to feel pain and yet be unable to speak to those caring for them [11]. This may be seen in patients with an endotracheal tube or a tracheostomy in situ, for example. Such patients will require very different pain assessments to those who are able to verbally self-report their pain.

More challengingly, patients in ICU frequently have reduced levels of consciousness. They can still experience pain but have impaired abilities to communicate it [12]. This may be seen, for example, in patients prescribed a sedative infusion to aid toleration of endotracheal intubation and mechanical ventilation. Such sedatives relieve agitation and distress but do not relieve pain. It is therefore possible to be deeply sedated but still in pain.

Reduced consciousness can also be seen in patients with a metabolic disturbance of any cause (such as respiratory failure due to chest sepsis), or with head injuries, and these illnesses can also be associated with pain [12]. Such patients will again require very different pain assessments to those who are fully conscious and able to communicate their pain.

\section{TOOLS TO ASSESS PAIN IN NON-VERBAL ICU PATIENTS}

In patients able to self-report their pain, Numerical Rating Scales (NRS) are often used [13]. These prompt a patient to rate the severity of their pain on a numerical scale from one (no pain) to ten (severe pain). In ICU patients unable to speak to those caring for them, an alternative Visual Analogue Scale (VAS) may be used [13]. This also asks patients to rate the severity of their pain, but by using a graphical version of the NRS. Patients may either move a marker device or simply point to the appropriate part of the scale. Simplified adaptations are available to improve patient comprehension. An example used in the care of infants and children, the Wong-Baker FACES Pain Rating Scale, is shown in Figure 1.

The VAS is well-established, and has been found to be reliable in assessing both acute and chronic pain. Such tools are particularly helpful because they standardise our descriptions of a patient's pain. Using standard numerical values rather than subjective descriptions like "severe" or "really bad" allows different doctors or nurses to semi-quantitatively compare pain assessments from day-to-day [9].

A study which introduced the VAS to an ICU found that this standardisation of pain assessment allowed better management of patient pain. At the introduction of VAS, $41 \%$ of ICU patients had pain greater than "three" in severity; after five weeks, less than $10 \%$ did [9]. 

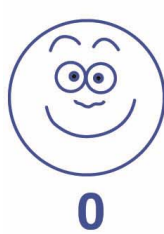

No

Hurt

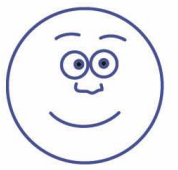

2

Hurts

Little Bit
Wong-Baker FACES ${ }^{T M}$ Pain Rating Scale

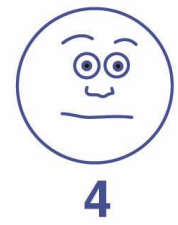

Hurts

Little More

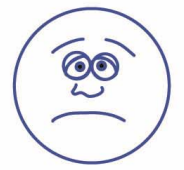

6

Hurts

Even More

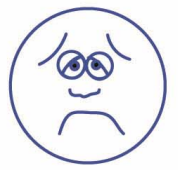

8

Hurts

Whole Lot

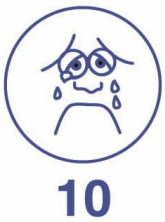

Hurts

Worst

(C)1983 Wong-Baker FACES TM Foundation. Visit us at www. WongBakerFACES.org Used with permission. Originally published in Whaley \& Wong's Nursing Care of Infants and Children. (CElsevier Inc.

Figure 1. The Wong-Baker FACES pain rating scale.

\section{TOOLS TO ASSESS PAIN IN UNCONSCIOUS OR SEDATED ICU PATIENTS}

Unfortunately, well-established and validated pain assessment tools such as NRS or VAS are often difficult or impossible to use in ICU as many patients have reduced conscious levels and/or are sedated. Alternative methods of pain assessment must therefore be used.

In unconscious patients, pain may be assessed through an examination of the patient's vital signs [14]. These assessments are based on the body's physiological responses to pain, such as tachycardia, tachypnoea, or hypertension. These parameters are often used by anaesthetists to guide analgesia in anaesthetized patients in theatre. It should be noted however that some studies have found that vital sign assessments of patient pain on ICU are not consistent with patient reports of pain $[14,15]$ (Table 3).

It may be difficult to assess patient pain using vital signs because ICU patients are often very unwell, with many potential causes for any change in their vital signs. Tachycardia may be due to pain, but could also be caused by fever or hypovolaemia. Because of this, some studies have suggested that these assessments of pain are unreliable [15]. New tools have therefore been developed to try to assess pain in unconscious patients more reliably.

An assessment of pain in unconscious ICU patients may be made using the Behavioural Pain Score (BPS) tool [16]. The BPS is an observational scale of patient behaviour, which allows an assessment of a patient's pain to be made by those caring for them (Table 4).

The BPS has three categories of behaviour: the patient's facial expression, the movement of their upper limbs, and their compliance with mechanical ventilation. The BPS provides descriptions of different behaviours which may be observed and assigns a score to each one. Higher scores are associated with greater pain. An overall pain score is then calculated, ranging from three (no pain) to twelve (worst possible pain).
Table 3. Potential clinical manifestations of pain.

\begin{tabular}{ll}
\hline Physiological system & Changes potentially associated with pain \\
\hline Respiratory & Tachypnoea \\
& Patient-ventilator dysynchrony \\
& Tachycardia \\
Cardiovascular & Bradycardia \\
& Hypertension \\
Neurology & Agitation \\
& Low mood \\
& Pupillary dilation \\
Other & Facial grimacing \\
& Sweating \\
& Crying \\
\hline
\end{tabular}

Table 4. The Behavioural Pain Scale.

\begin{tabular}{lll}
\hline Sub-scale & Description & Score \\
\hline \multirow{4}{*}{ Facial expression } & Relaxed & 1 \\
& Partially tightened & 2 \\
& Fully tightened & 3 \\
& Grimacing & 4 \\
& No movement & 1 \\
Upper limbs & Partially bent & 2 \\
& Fully bent with finger flexion & 3 \\
& Permanently retracted & 4 \\
Compliance with & Tolerating movement & 1 \\
& for most of the time & 2 \\
& Fighting ventilator & 3 \\
& Unable to control ventilation & 4
\end{tabular}


A number of studies have shown the BPS to be a reliable and valid method of assessing pain in ICU patients [27]. It has been found to be particularly helpful for recognising pain caused by routine procedures in ICU, such as turning or tracheal suctioning [17].

A similar behavioural scale called the Critical-Care Pain Observation Tool (CPOT) (Table 5) may also be used [18]. This is very similar to the BPS, but includes vocalisation as an additional category of behaviour. CPOT can therefore also be applied to patients who have been extubated after being mechanically ventilated.

ICU nurses using the CPOT in practice reported that it was helpful for nursing practice, provided them with a common language, and standardised their assessments of pain [19].

\section{CHANGES IN ICU PRACTICE}

In ICU patients with impaired self-reporting due to sedation, changes in sedation practice itself may improve the recognition and management of pain [20].

Sedative infusions are often prescribed to relieve agitation and distress in ICU patients. The depth of patient sedation is measured using validated sedation scoring systems such as the Ramsay or Richmond AgitationSedation Scales (RASS) [21].

Traditionally, ICU patients have been continuously, deeply, sedated (Ramsay score 5 to 6 ; RASS -4 to -5 ). It has been noted, however, that continuous deep sedation

Table 5. The Critical Care Pain Observation Tool.

\begin{tabular}{|c|c|c|}
\hline Sub-scale & Description & Score \\
\hline & Relaxed, neutral & 0 \\
\hline \multirow[t]{3}{*}{ Facial expression } & Tense & 1 \\
\hline & Grimacing & 2 \\
\hline & Absence of movements & 0 \\
\hline \multirow[t]{3}{*}{ Body movements } & Protection & 1 \\
\hline & Restlessness & 2 \\
\hline & Relaxed & 0 \\
\hline \multirow[t]{3}{*}{ Muscle tension } & Tense, rigid & 1 \\
\hline & Very tense or rigid & 2 \\
\hline & Tolerating ventilator or movement & 0 \\
\hline \multirow[t]{3}{*}{$\begin{array}{l}\text { Compliance with } \\
\text { ventilation }\end{array}$} & Coughing but tolerating & 1 \\
\hline & Fighting ventilator & 2 \\
\hline & Talking in normal tone or no sound & 0 \\
\hline \multirow[t]{2}{*}{$\begin{array}{l}\text { Vocalisation } \\
\text { (extubated patients) }\end{array}$} & Sighing, moaning & 1 \\
\hline & Crying out, sobbing & 2 \\
\hline
\end{tabular}

prolongs a patient's requirement for mechanical ventilation, their time in ICU, and their time in hospital [22]. It also makes routine patient assessments more difficult. The practice of interrupting this continuous sedation daily, until the patient is awake (Ramsay score 2 to 3; RASS 0 to -1), may be beneficial [23].

Some studies have found that such "sedation holds" can shorten a patient's requirement for ventilation, their time in ICU, and their time in hospital [24]. Sedation holds have also been noted to improve our ability to undertake routine patient assessments, including an assessment of patient pain [23,24] (Table 6).

Another approach to patient sedation in ICU is the use of "analgo-sedation". Traditionally, ICU patients have been treated using a "sedation-analgesia" model [25]. Patients are given constant sedatives to relieve anxiety or distress, with extra analgesia given to relieve any pain. The analgo-sedation model, however, uses a drug regime that prioritises analgesia first, together with lighter, interrupted, sedation (Ramsay score 2 to 3 ; RASS 0 to -1 ) [25] (Table 7).

In one study, the analgo-sedation approach increased the proportion of pain-free ICU patients from $56.8 \%$ to

Table 6. The Ramsay Scale of Sedation.

\begin{tabular}{ll}
\hline Level of activity & Score \\
\hline Anxious or restless & 1 \\
Cooperative, orientated, and calm & 2 \\
Responds to commands only & 3 \\
Brisk response to stimulus & 4 \\
Sluggish response to stimulus & 5 \\
No response to stimulus & 6 \\
\hline
\end{tabular}

Table 7. The Richmond Agitation-Sedation Scale (RASS).

\begin{tabular}{ll}
\hline Term & Score \\
\hline Combative & +4 \\
Very agitated & +3 \\
Agitated & +2 \\
Restless & +1 \\
Alert and calm & 0 \\
Drowsy & -1 \\
Light sedation & -2 \\
Moderate sedation & -3 \\
Deep sedation & -4 \\
Unrousable & -5 \\
\hline
\end{tabular}


$82.7 \%$ [25]. It also significantly reduced patient distress, anxiety, and agitation.

A recent single-centre study suggests that a "no-sedation" model may improve clinical outcomes in critically ill patients, without increasing the incidence of adverse psychological effects. In this study, patients were given bolus analgesia alone, with no sedation unless deemed clinically essential. This approach was associated with a reduction in the duration of mechanical ventilation, together with a reduction in ICU and hospital lengths of stay [26]. More research is needed to see whether this approach improves the recognition and management of patient pain [26].

\section{CONCLUSIONS}

Review of the literature suggests that patients on ICU often suffer from undertreated and unrecognised pain, with potentially serious physical and psychological effects. This is likely because the accurate assessment of pain in ICU is very difficult. ICU patients are less able to communicate their pain to us than non-ICU patients, and are frequently sedated.

A number of tools have been developed to improve our assessment of pain. This includes the visual analogue pain scale (VAS) for patients unable to speak, and the behavioural pain scale (BPS) and critical care pain observation tool (CPOT) for patients who have reduced consciousness levels, or are sedated (Table 8).

Challenging the concept that a sedated, unresponsive patient is pain free is essential. Improvements in sedation practice - moving from the traditional use of sedatives to achieve constant deep sedation, to daily interrupted sedation, with sedation regimes targeting analgesia rather than simply sedation - is likely to improve both the recognition and treatment of pain.

\section{ACKNOWLEDGEMENTS}

Thanks to Dr. David Garry, Specialist Registrar in Anaesthetics \& Intensive Care, Oxford; and Dr. John Griffiths, Consultant in Anaesthetics \& Intensive Care, Oxford.

Table 8. Tools for improving pain recognition.

\begin{tabular}{ll}
\hline Patient's condition & Tools \\
\hline $\begin{array}{l}\text { Conscious and able } \\
\text { to verbalise }\end{array}$ & $\begin{array}{l}\text { Numerical Rating Scale (NRS) } \\
\text { of pain severity }\end{array}$ \\
$\begin{array}{l}\text { Conscious but unable } \\
\text { to verbalise }\end{array}$ & $\begin{array}{l}\text { Visual Analogue Scale (VAS) } \\
\text { of pain severity }\end{array}$ \\
$\begin{array}{l}\text { Assessment of patient's vital signs } \\
\text { consciousness/sedated }\end{array}$ & $\begin{array}{l}\text { Behavioural Pain Scale (BPS) } \\
\text { Critical Care Pain Observation Tool } \\
\text { (CPOT) }\end{array}$ \\
\hline
\end{tabular}

\section{SEARCH STRATEGY}

Evidence for this review was provided by a search of the Medline/ Pubmed, Embase, Google, Google Scholar, and Cochrane Library, and relevant guidelines, up to August 2012.

A Pubmed and Google Scholar search were completed from August 1990 to 2012 looking for the following terms in the title or abstract: "pain intensive care unit (ICU/ITU)", "causes of pain ICU", "pain assessment ICU”, "pain recognition ICU”, and "pain management ICU”.

\section{REFERENCES}

[1] Gelinas, C. (2007) Management of pain in cardiac surgery ICU patients: Have we improved over time? Intensive and Critical Care Nursing, 23, 298-303. doi:10.1016/j.iccn.2007.03.002

[2] Sessler, C.N. (2009) Progress towards eliminating inadequately managed pain in the ICU through interdisciplinary care. Chest, $\mathbf{1 3 5}, 894-896$. doi:10.1378/chest.08-2834

[3] Jones, C., Backman, C., Capuzzo, M., et al. (2007) Precipitants of post-traumatic stress disorder following intensive care: A hypothesis generating study of diversity in care. Intensive Care Medicine, 33, 978-985. doi:10.1007/s00134-007-0600-8

[4] Cousins, M.J., Brennan, F. and Carr, D.B. (2004) Pain relief: A universal human right. Pain, 112, 1-4. doi:10.1016/i.pain.2004.09.002

[5] Copp, L.A. (2006) An ethical responsibility for pain management. Journal Advanced Nursing, 55, 1-3. doi:10.1111/j.1365-2648.2006.03892 1.x

[6] IASP Task Force on Taxonomy, IASP Taxonomy (2011). http://www.iasp-pain.org

[7] Herr, K., Coyne, P., Key, T., et al. (2006) Pain assessment in the nonverbal patient: Position statement with clinical practice recommendations. Pain Management Nursing, 7, 44-52. doi:10.1016/j.pmn.2006.02.003

[8] Pasero, C., Puntillo, K., Li, D., et al. (2009) Structured approaches to pain management in the ICU. Chest, 135, 1665-1672. doi:10.1378/chest.08-2333

[9] Erdek, M.A. and Pronovost, P.J. (2004) Improving assessment and treatment of pain in the critically ill. International Journal for Quality in Health Care, 16, 59-64. doi:10.1093/intqhe/mzh010

[10] Puntillo, K., Pasero, C. and Li, D. (2009) Evaluation of pain in ICU patients. Chest, 135, 1069-1074. doi:10.1378/chest.08-2369

[11] Paulson-Conger, M., Leske, J. and Maidl, C. (2010) Comparison of two different pain assessment tools in nonverbal critical care patients. Pain Management Nursing, 12, 218-224. doi:10.1016/j.pmn.2010.05.008

[12] Pudas-Tahka, S., Axelin, A., Lund, V., et al. (2009). Pain assessment tools for unconscious or sedated intensive care patients: A systematic review. Journal Advanced Nursing, 65, 946-956. doi:10.1111/j.1365-2648.2008.04947.x

[13] Jensen Hjermstad, M., Fayers, P.M., Haugen, D.F., et al. 
(2011) Studies comparing numeric rating scales, verbal rating scales, and visual analogue scales for assessment of pain intensity in adults: A systematic literature review. Journal Pain Symptom Management, 41, 1073-1093. doi:10.1016/j.jpainsymman.2010.08.016

[14] Arbour, C. and Gelinas, C. (2010) Are vital signs valid indicators for the assessment of pain in postoperative cardiac surgery ICU adults? Intensive and Critical Care Nursing, 26, 83-90. doi:10.1016/j.iccn.2009.11.003

[15] Gelinas, C., Tousignant-Laflamme, Y. and Tanquay, A. (2011) Exploring the validity of the bispectral index, the critical-care pain observation tool and vital signs for the detection of pain in sedated and mechanically ventilated critically ill adults: A pilot study. Intensive and Critical Care Nursing, 27, 46-52. doi:10.1016/j.iccn.2010.11.002

[16] Ahlers, S.J., van der Veen, A.M., van Dijk, M., et al. (2010) The use of the behavioural pain scale to assess pain in conscious sedated patients. Anaesthesia and Analgesia, 110, 127-133. doi:10.1213/ANE.0b013e3181c3119e

[17] Aissaoui, Y., Zeggwagh, A.A., Zekraoui, A., et al. (2005) Validation of a behavioral pain scale in critically ill, sedated, and mechanically ventilated patients. Anaesthesia and Analgesia, 101, 1470-1476. doi:10.1213/01.ANE.0000182331.68722.FF

[18] Tousignant-Laflamme, Y., Bourgault, P., Gelinas, C., et al. (2010) Assessing pain behaviours in health subjects using the critical-care pain observation tool (CPOT): A pilot study. Journal Pain, 11, 983-987. doi:10.1016/j.jpain.2010.01.266

[19] Gelinas, C., Fillion, L., Puntillo, K., et al. (2006) Valida- tion of the critical-care observation tool in adult patients. American Journal of Critical Care, 15, 420-427.

[20] Gelinas, C. (2009) Nurses evaluation of the feasibility and the clinical utility of the critical-care pain observation tool. Pain Management Nursing, 11, 115-125. doi:10.1016/j.pmn.2009.05.002

[21] Woien, H., Stubhaug, A. and Bjork, I.T. (2011) Analgesia and sedation of mechanically ventilated patients-A national survey of clinical practice. Acta Anaesthesiologica Scandinavica, 56, 23-29. doi:10.1111/j.1399-6576.2011.02524.x

[22] Sessler, C.N., Grap, M.J. and Ramsay, M.A.E. (2008) Evaluating and monitoring analgesia and sedation in the intensive care unit. Critical Care, 12, S2.

[23] Selles, C. and Wai, M.C.G.T.J. (2010) Conscious sedation in the intensive care unit: The future? Erasmus Journal of Medicine, 1, 26-29.

[24] Kress, J.P., Pohlman, A.S., O’Connor, M.D., et al. (2000) Daily interruption of sedative infusions in critically ill patients undergoing mechanical ventilation. New England Journal of Medicine, 342, 1471-1477. doi:10.1056/NEJM200005183422002

[25] Egerod, I., Jensen, M.B., Herling, S.F., et al. (2010) Effect of an analgo-sedation protocol for neurointensive patients: A two-phase interventional non-randomized pilot study. Critical Care, 14, R71.

[26] Strom, T., Martinussen, T. and Toft, P. (2010) A protocol of no-sedation for critically ill patients requiring mechanical ventilation: A randomized trial. Lancet, 375, 475-480. doi:10.1016/S0140-6736(09)62072-9 